\section{Prevalência de sibilância e fatores associados em crianças indígenas Guarani hospitalizadas por doença respiratória aguda no Sul e Sudeste do Brasil}

\author{
Prevalence of wheezing and associated factors \\ in Guarani indigenous children hospitalized for \\ acute respiratory infections in Southern and \\ Southeastern Brazil
}

\author{
Prevalencia de sibilancia y factores asociados \\ en niños indígenas guaraníes hospitalizados \\ por enfermedad respiratoria aguda en el \\ sur y sureste de Brasil
}

Abstract

Prevalence of wheezing was studied in Guarani indigenous children hospitalized for acute lower respiratory tract infections in Southern and Southeastern Brazil, recruited by a surveillance routine established in villages from May 2007 to June 2008. Data were obtained from hospital records. Crude and adjusted prevalence ratios for wheezing were estimated according to categories of target variables, using Poisson regression with robust variance. Prevalence of wheezing was $58.1 \%$ (136/234). Risk factors that remained significantly associated with wheezing in the final model were: age bracket, inversely associated ( 0 11 months: reference; 24-35 months: 0.63, 95\%CI: 0.40-0.99); hospitalization in the autumn (summer: reference; autumn: 1.58, 95\% CI: 1.05-2.40); dyspnea (1.41, 95\%CI: 1.09-1.83); chest indrawing (1.42, 95\%CI: 1.16-1.73); crackles (1.43, 95\%CI: 1.09-1.87). The results show a high disease burden related to wheezing and suggest the phenotype of early-onset persistent wheezing related to recurrence of viral respiratory infections.

Respiratory Sounds; Pneumonia; South American Indians; Child; Prevalence
Patrícia Gomes de Souza 1 Andrey Moreira Cardoso 2 Clemax Couto Sant'Anna 1

\section{Resumo}

Realizou-se estudo de prevalência de sibilância em crianças indígenas Guarani hospitalizadas por infecção respiratória aguda baixa no Sul e Sudeste do Brasil, recrutadas a partir de um sistema de vigilância implantado nas aldeias entre maio de 2007 e junho de 2008. Os dados foram extraídos por revisão dos prontuários hospitalares. Estimaram-se razões de prevalência de sibilância brutas e ajustadas segundo categorias das variáveis exploratórias, por regressão de Poisson com ajuste para variância robusta. A prevalência de sibilância foi de 58,1\% (136/234). Permaneceram significativamente associadas à sibilância no modelo final: faixa etária, inversamente associada (0-11 meses: referência; 24-35 meses: 0,63, IC95\%: 0,40-0,99); internação no outono (verão: referência; outono: 1,58, IC95\%: 1,05-2,40); e sinais clínicos de dispneia (1,41, IC95\%: 1,09-1,83), tiragem (1,42, IC95\%: 1,16-1,73) e estertor (1,43, IC95\%: 1,09-1,87). Os resultados revelam elevada carga de morbidade por sibilância e sugerem o fenótipo de sibilância persistente de início precoce, relacionada à recorrência de infecções respiratórias virais.

Sons Respiratórios; Pneumonia; Índios Sul-Americanos; Criança; Prevalência 


\section{Introdução}

A sibilância é um dos sintomas respiratórios mais comuns na infância, manifestando-se frequentemente antes de um ano de idade 1 . A morbimortalidade por causas respiratórias relacionadas à sibilância é elevada, sendo mais vulneráveis os lactentes de populações socieconomicamente menos favorecidas 2 , nos quais o primeiro episódio de sibilância pode resultar de inflamação dos bronquíolos de etiologia viral, a bronquiolite viral aguda ${ }^{3}$. Na América Latina, a sibilância em lactentes tem sido frequentemente relacionada à exposição precoce e continuada a agentes inalantes do ambiente e às infecções respiratórias virais, podendo evoluir com complicações, como a pneumonia 4 .

São descritos diversos fenótipos de sibilância decorrentes da interação entre fatores genéticos e ambientais: (1) sibilância transitória, que ocorre nos três primeiros anos de idade e geralmente está associada à prematuridade e ao tabagismo passivo; (2) sibilância persistente de início precoce, com primeiro episódio antes dos três anos de idade e recorrência associada a infecções respiratórias agudas virais, sem evidência e história familiar de atopia; e (3) sibilância persistente de início tardio ou asma, como expressão de uma via aérea patogênica decorrente de atopia 5 .

Os povos indígenas encontram-se entre os grupos sociais mais marginalizados na América Latina e no Caribe ${ }^{6}$. No Brasil, assim como em outros países, o perfil de morbimortalidade desses povos é marcado por doenças infecciosas, parasitárias e carenciais, particularmente na população infantil, com destaque para as infecções respiratórias agudas (IRA), em sua maioria preveníveis ou reduzíveis por intervenções disponíveis no Sistema Único de Saúde (SUS) 6,7,8,9,10,11,12,13.

Em recente estudo sobre doença respiratória aguda em indígenas Guarani residentes em 83 aldeias no Sul e Sudeste do Brasil, verificouse que $71,9 \%$ das hospitalizações com causas definidas em $<5$ anos de idade foram devidas às infecções respiratórias agudas baixas (IRAB), sendo o broncoespasmo, a bronquiolite viral aguda e a asma as principais causas secundárias de diagnóstico ${ }^{7}$. Nessa mesma população e no mesmo período, a taxa de mortalidade em $<5$ anos e a taxa de mortalidade infantil corresponderam a 44,5 óbitos por mil nascidos vivos e a 29,6 óbitos por mil nascidos vivos, respectivamente. A mortalidade por IRAB $(<5$ anos: 24,7 por mil nascidos vivos; $<1$ ano: 17,3 por mil nascidos vivos) foi a maior entre as causas específicas de mortalidade, superando em pelo menos duas vezes as taxas de mortalidade por diarreia e por demais causas. IRAB e diarreia em conjunto, responderam por $100 \%$ das causas de morte em crianças de 1 a 4 anos de idade e a $90 \%$ das mortes infantis 8 .

$\mathrm{Na}$ investigação dos fatores de risco para hospitalização de crianças Guarani por IRAB, verificou-se associação significativa com baixa renda estável per capita familiar, elevado número de moradores no domicílio, baixa idade materna, exposição à poluição intradomicilar e baixo peso ao nascer, evidenciando as precárias condições de vida às quais a população Guarani está submetida 14 .

O estudo sobre doença respiratória aguda nos Guarani produziu as primeiras evidências sobre seus determinantes em povos indígenas do Brasil e demonstrou que essa é a principal causa de morbidade e mortalidade nas crianças da etnia. Os resultados do componente morbidade hospitalar do estudo sugerem que a hiperreatividade brônquica esteja implicada na fisiopatogenia das hospitalizações Guarani 7. Portanto, este estudo visa descrever a prevalência de sibilância e investigar os fatores associados nas crianças Guarani < 5 anos hospitalizadas por IRAB no Sul e Sudeste do Brasil, no período de maio de 2007 a junho de 2008, ocasião em que se implantou um sistema de vigilância das hospitalizações para a operacionalização do estudo sobre doença respiratória aguda em crianças da etnia.

\section{Métodos}

Trata-se de estudo de prevalência de sibilância e fatores associados em crianças indígenas Guarani hospitalizadas, residentes em 83 aldeias da etnia dispersas na faixa litorânea que se estende do sul do Estado do Rio de Janeiro ao Estado de Santa Catarina e em todo o Estado do Rio Grande do Sul. Em geral, as aldeias vivem em situação de vulnerabilidade tanto por condições ambientais, quanto por situações socioeconômicas e habitacionais precárias 14 . A atenção primária à saúde nas aldeias é prestada por Equipes Multidisciplinares de Saúde Indígena (EMSI) sob responsabilidade do Subsistema de Atenção à Saúde dos Povos Indígenas, sendo a complementariedade das ações de saúde em outros níveis de complexidade, realizada pelas redes municipais e estaduais de saúde.

Os dados para esta análise são provenientes de uma série de hospitalizações de crianças indígenas Guarani por IRAB, ocorridas entre 1o de maio de 2007 e 30 de junho de 2008. Os casos foram recrutados a partir de um sistema de vigilância coordenado por um dos pesquisadores e conduzido pelos enfermeiros das EMSI, implantado especificamente para realização de um 
estudo caso-controle sobre fatores de risco para hospitalização de crianças indígenas da etnia por IRAB 14. Estabeleceu-se uma rotina de vigilância visando à notificação semanal, positiva ou negativa, das hospitalizações por IRAB em $<5$ anos. Na eventual falha de notificação, a coordenação da pesquisa realizava contato telefônico a fim de confirmar a ocorrência ou não de hospitalização. Essas ações foram complementadas por visitas sistemáticas aos enfermeiros responsáveis pelas notificações nas aldeias e por busca ativa de hospitalizações e revisão de prontuários nos 49 hospitais onde ocorreram internações Guarani 15. Procedeu-se à extração de dados a partir da revisão dos registros dos prontuários de internação, por meio da aplicação de um formulário padronizado (Formulário de Extração de Dados Hospitalares), preenchido pelo médico coordenador da pesquisa. Detalhes metodológicos adicionais estão descritos em outros artigos 7,8,14.

Foram analisadas variáveis: (a) demográficas e climáticas - idade, sexo, região de residência, estações do ano de nascimento e de internação (20 de março a 20 de junho, outono; 21 de junho a 22 de setembro, inverno; 23 de setembro a 21 de dezembro, primavera; 22 de dezembro a 19 de março, verão. Observatório Astronômico de Uberlândia. Estações do ano - 2003. http://www. silvestre.eng.br/astronomia/fenomenos/estaco es/2003/, acessado em 13/Dez/2013); (b) clínicas - tosse, febre, dispneia, taquipneia, estertores, tiragem, sibilância, sinais de gravidade, hipótese diagnóstica (IRA pneumonia e IRA não pneumonia); (c) laboratoriais - contagem de eosinófilos no sangue periférico e gasometria arterial; (d) radiológicas - infiltrados alveolar e intersticial, hiperinsuflação pulmonar e retificação dos arcos costais; (e) terapêuticas - uso de antibiótico; e (f) evolução - duração da internação, reinternação no mesmo episódio, novos episódios de internação e óbito. Variáveis clínicas sem registro no prontuário foram definidas como ausentes na criança. Para o diagnóstico, foram considerados dois critérios: o diagnóstico médico registrado no prontuário e o diagnóstico de um pneumopediatra (revisor), a partir dos dados extraídos do prontuário, com base em um critério clínico-radiológico-laboratorial pré-definido estabelecido a partir de revisão da literatura 15 .

Elaborou-se um banco de dados no programa Epi Info, versão 3.5.1 (Centers for Disease Control and Prevention, Atlanta, Estados Unidos) e as análises foram conduzidas no programa Stata 10 (Stata Corp., College Station, Estados Unidos). Foram descritas as distribuições de frequência de hospitalizações e as prevalências de sibilância segundo categorias das variáveis exploratórias. Em seguida, foram estimadas razões de prevalências brutas e ajustadas de sibilância por regressão de Poisson com ajuste para variância robusta 16 , com respectivos intervalos de $95 \%$ de confiança (IC95\%). Para a análise multivariada, utilizou-se um modelo hierárquico cujos níveis de análise foram, do mais distal para o mais proximal: nível 1 - região de residência; nível 2 - condições demográficas e climáticas; nível 3 - condições clínicas; e nível 4 - padrões radiológicos e laboratoriais. As associações de sibilância com as variáveis ligadas ao diagnóstico, tratamento e desfecho foram consideradas separadamente das demais, tendo em vista sua relação de consequência e não de causa com o desfecho.

A variável região de residência, pertencente ao nível mais distal, foi a primeira a entrar na análise multivariada. Nos demais níveis, as variáveis foram incluídas na análise multivariada correspondente ao seu nível, juntamente com as variáveis retidas nos níveis anteriores, aplicandose o método backward para excluir as de menor significância, até se manterem no modelo final apenas aquelas com $\mathrm{p}<0,20$. Assim, cada variável mantida no modelo final encontra-se ajustada pelas demais variáveis retidas do mesmo nível de análise e pelas variáveis retidas nos níveis de análise anteriores.

\section{Aspectos éticos}

O projeto foi aprovado pelo Comitê de Ética em Pesquisa da Escola Nacional de Saúde Pública Sergio Arouca, Fundação Oswaldo Cruz (Ensp/ Fiocruz, parecer no $130 / 2005)$ e pela Comissão Nacional de Ética em Pesquisa (CONEP, parecer no 154/2006). Obteve-se autorização da Fundação Nacional do Índio (autorização no 23/ CGEP/2007) para entrada em Terra Indígena a fim de realizar pesquisa científica, mediante assinatura dos termos de consentimento livre e esclarecido coletivos pelas lideranças indígenas de cada uma das aldeias participantes.

\section{Resultados}

Foram analisadas 234 hospitalizações por IRAB de crianças Guarani entre 0 e 59 meses de idade, o que corresponde a $68,8 \%$ do total de hospitalizações ocorridas no período. Esse tamanho de amostra confere um poder de $70 \%$ para detectar desigualdades nas prevalências se as mesmas forem inferiores a $41,9 \%$ ou superiores a $58,1 \%$, a um nível de significância de $5 \%$. A média de idade foi de 15,4 \pm 13,0 meses (mediana $=11$ meses). Um quarto das crianças tinha menos de seis meses e $75 \%$ delas tinham menos de 21 meses de idade. Do total de hospitalizações analisadas, 
$136(58,1 \%)$ possuíam registro de sibilância no prontuário.

A distribuição de frequência das hospitalizações e as prevalências de sibilância segundo região de residência, características demográficas e climáticas encontram-se na Tabela 1. A frequência de crianças hospitalizadas foi maior no Sudeste $(67,9 \%)$, foi similar entre os sexos (feminino: $50,8 \%$ e concentrou-se na faixa etária mais baixa (<12 meses: $50,8 \%$ ). As proporções de nascimentos e internações foram mais elevadas no outono (20 de março a 20 de junho). Na análise bruta, as variáveis região de residência, sexo, faixa etária e estação de internação apresentaram nível de significância $\mathrm{p}<0,20$, tendo sido incluídas na regressão multivariada.

Tosse, febre, taquipneia, dispneia e estertores apresentaram frequências que variaram de $52,1 \%$ (taquipneia) a $80,8 \%$ (tosse) (Tabela 2). Por outro lado, sinais e sintomas de gravidade da doença tiveram frequências inferiores a 5\%. Taquip- neia, dispneia, tiragem e estertor apresentam associação direta e estatisticamente significativa com sibilância na análise bruta, ao passo que tosse mostrou-se inversamente associada. Além dessas, febre e esforço respiratório apresentaram valor de $\mathrm{p}<0,20$, sendo também incluídas na regressão multivariada.

Dos prontuários analisados, 133 (56,7\%), 85 (36,3\%) e 202 (86,3\%) não possuíam registro de realização de radiografia de tórax, contagem de eosinófilos e saturação periférica de oxigênio, respectivamente. Em decorrência da baixa frequência de registro de gasometria nos prontuários, essa variável foi excluída das análises. Entre os prontuários com registro de radiografia de tórax, apenas três $(1,3 \%)$ estavam normais ou com alterações não relacionadas à IRAB. Entre os com alterações relacionadas à IRAB, a frequência de infiltrado intersticial associado ou não a hiperinsuflação e/ou retificação de arcos costais foi 9\%. A frequência de eosinofilia foi de 14,8\% (Tabela 3).

Tabela 1

Frequência de hospitalização e prevalência e razões de prevalência de sibilância segundo variáveis região de residência, demográficas e climáticas.

\begin{tabular}{|c|c|c|c|c|c|c|c|c|}
\hline \multirow[t]{2}{*}{ Variáveis } & \multicolumn{3}{|c|}{ Hospitalizações } & \multicolumn{3}{|c|}{ Prevalência de sibilância } & \multirow{2}{*}{$\mathrm{RP}_{\text {robusto }}$} & \multirow[t]{2}{*}{ IC95\% } \\
\hline & $\mathrm{n}$ & $\%$ & IC95\% & $\mathrm{n}$ & $\%$ & IC95\% & & \\
\hline \multicolumn{9}{|l|}{ Nível 1} \\
\hline Região de residência & & & & & & & $p=0,110$ & \\
\hline Sudeste & 159 & 67,9 & $61,9-74,0$ & 87 & 54,7 & $46,9-62,5$ & 1,00 & \\
\hline Sul & 75 & 32,1 & $26,0-38,0$ & 49 & 65,3 & $54,5-76,2$ & 1,19 & $0,96-1,48$ \\
\hline \multicolumn{9}{|l|}{ Nível 2} \\
\hline Sexo & & & & & & & $p=0,174$ & \\
\hline Feminino & 119 & 50,8 & $44,4-57,3$ & 64 & 53,8 & $44,7-62,8$ & 1,00 & \\
\hline Masculino & 115 & 49,2 & $42,7-55,6$ & 72 & 62,6 & $53,7-71,5$ & 1,16 & $0,93-1,45$ \\
\hline Faixa etária (meses) & & & & & & & $p=0,150$ & \\
\hline $0-11$ & 119 & 50,8 & $44,4-57,3$ & 77 & 64,7 & $56,0-73,3$ & 1,00 & \\
\hline $12-23$ & 62 & 26,5 & $20,8-32,2$ & 32 & 51,6 & $39,1-64,1$ & 0,80 & $0,60-1,05$ \\
\hline $24-35$ & 27 & 11,5 & $7,41-15,6$ & 12 & 44,4 & $25,6-63,3$ & 0,69 & $0,44-1,07$ \\
\hline $36-59$ & 26 & 11,2 & $7,05-15,1$ & 15 & 57,7 & $38,6-76,8$ & 0,89 & $0,62-1,27$ \\
\hline Estação de nascimento & & & & & & & $p=0,134$ & \\
\hline Verão & 38 & 16,3 & $11,5-21,0$ & 21 & 55,3 & $39,3-71,2$ & 1,00 & \\
\hline Outono & 89 & 38,0 & $31,7-44,3$ & 47 & 52,8 & $42,4-63,2$ & 0,95 & $0,67-1,35$ \\
\hline Inverno & 56 & 23,9 & $18,4-29,4$ & 34 & 60,7 & $47,8-73,6$ & 1,10 & $0,77-1,57$ \\
\hline Primavera & 51 & 21,8 & $16,5-27,1$ & 34 & 66,7 & $53,6-79,7$ & 1,21 & $0,85-1,70$ \\
\hline Estação de internação & & & & & & & $p=0,362$ & \\
\hline Verão & 32 & 13,8 & $9,24-18,1$ & 14 & 43,7 & $26,4-61,1$ & 1,00 & \\
\hline Outono & 81 & 34,6 & $28,5-40,7$ & 52 & 64,2 & $53,7-74,7$ & 1,47 & $0,96-2,25$ \\
\hline Inverno & 57 & 24,3 & $18,8-29,9$ & 30 & 52,6 & $39,6-65,7$ & 1,20 & $0,75-1,91$ \\
\hline Primavera & 64 & 27,3 & $21,6-33,1$ & 40 & 62,5 & $50,5-74,4$ & 1,43 & $0,92-2,21$ \\
\hline
\end{tabular}

IC95\%: intervalo de 95\% de confiança; RP: razão de prevalência. 
Frequência de hospitalização e prevalência e razões de prevalência de sibilância segundo características clínicas.

\begin{tabular}{|c|c|c|c|c|c|c|c|c|}
\hline \multirow[t]{2}{*}{ Variáveis } & \multicolumn{3}{|c|}{ Hospitalizações } & \multicolumn{3}{|c|}{ Prevalência de sibilância } & \multirow{2}{*}{$\mathrm{RP}_{\text {robusto }}$} & \multirow[t]{2}{*}{ IC95\% } \\
\hline & $\mathrm{n}$ & $\%$ & IC95\% & $\mathrm{n}$ & $\%$ & IC95\% & & \\
\hline \multicolumn{9}{|l|}{ Nível 3} \\
\hline Tosse & & & & & & & $p=0,008$ & \\
\hline Não & 45 & 19,2 & $14,1-24,3$ & 33 & 73,3 & $60,3-86,3$ & 1,00 & \\
\hline $\operatorname{Sim}$ & 189 & 80,8 & $75,7-85,8$ & 103 & 54,5 & $47,3-61,6$ & 0,74 & $0,60-0,93$ \\
\hline Febre & & & & & & & $p=0,142$ & \\
\hline Não & 79 & 33,8 & $27,6-39,9$ & 51 & 64,6 & $53,9-75,2$ & 1,00 & \\
\hline Sim & 155 & 66,2 & $60,1-72,3$ & 85 & 54,8 & $46,9-62,7$ & 0,85 & $0,68-1,06$ \\
\hline Taquipneia & & & & & & & $p=0,009$ & \\
\hline Não & 112 & 47,9 & $41,4-54,3$ & 55 & 49,1 & $39,8-58,4$ & 1,00 & \\
\hline Sim & 122 & 52,1 & $45,7-58,6$ & 81 & 66,4 & $57,9-74,8$ & 1,35 & $1,08-1,70$ \\
\hline Dispneia & & & & & & & $p=0,001$ & \\
\hline Não & 81 & 34,6 & $28,5-40,7$ & 34 & 42,0 & $31,1-52,8$ & 1,00 & \\
\hline $\operatorname{Sim}$ & 153 & 65,4 & $59,2-71,5$ & 102 & 66,7 & $59,1-74,2$ & 1,59 & $1,20-2,10$ \\
\hline Esforço respiratório & & & & & & & $p=0,168$ & \\
\hline Não & 208 & 88,9 & $84,8-93,0$ & 118 & 56,7 & $49,9-63,5$ & 1,00 & \\
\hline $\operatorname{Sim}$ & 26 & 11,1 & $7,05-15,2$ & 18 & 69,2 & $51,3-87,1$ & 1,22 & $0,92-1,62$ \\
\hline Tiragem & & & & & & & $p=0,000$ & \\
\hline Não & 160 & 68,4 & $62,4-74,4$ & 78 & 48,7 & $40,9-56,5$ & 1,00 & \\
\hline Sim & 74 & 31,6 & $25,6-37,6$ & 58 & 78,4 & $68,9-87,8$ & 1,61 & $1,32-1,96$ \\
\hline Estertores & & & & & & & $p=0,006$ & \\
\hline Não & 72 & 30,8 & $24,8-36,7$ & 31 & 43,1 & $31,5-54,6$ & 1,00 & \\
\hline Sim & 162 & 69,2 & $63,3-75,2$ & 105 & 64,8 & $57,4-72,2$ & 1,50 & $1,13-2,01$ \\
\hline Recusa alimentar & & & & & & & $p=0,228$ & \\
\hline Não & 225 & 96,1 & $93,7-98,6$ & 133 & 59,1 & $52,6-65,6$ & 1,00 & \\
\hline Sim & 9 & 3,9 & $1,36-6,33$ & 3 & 33,3 & $2,3-64,3$ & 0,56 & $0,22-1,43$ \\
\hline Batimento de asa do nariz & & & & & & & $p=0,329$ & \\
\hline Não & 228 & 97,4 & $95,4-99,5$ & 134 & 58,8 & $52,3-65,2$ & 1,00 & \\
\hline Sim & 6 & 2,6 & $0,52-4,60$ & 2 & 33,3 & $4,7-71,3$ & 0,57 & $0,18-1,77$ \\
\hline Complicações & & & & & & & $p=0,532$ & \\
\hline Não & 218 & 93,2 & $90,0-96,4$ & 128 & 58,7 & $52,1-65,3$ & 1,00 & \\
\hline Sim & 16 & 6,8 & $3,60-10,1$ & 8 & 50,0 & $25,3-74,7$ & 0,85 & $0,51-1,41$ \\
\hline
\end{tabular}

IC95\%: intervalo de 95\% de confiança; RP: razão de prevalência.

As associações de sibilância com padrão radiológico e eosinofilia não apresentaram significância estatística na análise bruta.

No modelo multivariado, a sibilância mostrou-se inversamente e significativamente associada à faixa etária da criança. Além disso, a sibilância foi significativamente mais prevalente nas crianças hospitalizadas no outono e naquelas com registro em prontuário de dispneia, tiragem e estertor. Tosse e taquipneia perderam significância estatística após ajuste por variáveis de confundimento (Figura 1).

A hipótese diagnóstica de pneumonia foi registrada em $82,1 \%$ dos prontuários, sendo esta proporção mais baixa pela classificação do revisor $(67,5 \%)$ (Tabela 4). Na quase totalidade das hospitalizações $(96,2 \%)$, houve prescrição de antibiótico. A média de tempo de permanência hospitalar foi de 7,6 (DP = 6,3) dias. O tempo de permanência hospitalar variou de um a 63 dias, sendo que em $8,1 \%$ das hospitalizações, foi menor que três dias. Em 25\%, 50\% e 75\% das hospitalizações, esses tempos corresponderam a 4, 6 e 9 dias, respectivamente. A permanência hospitalar foi de até 10 dias em 80,3\% das hospitalizações. Verificaram-se reinternação da criança pelo mesmo episódio em $2,1 \%$ dos casos e reinternação da mesma criança por outro episódio em $24,8 \%$ 
Frequência de hospitalização e prevalência e razões de prevalência de sibilância segundo características radiológicas e laboratoriais.

\begin{tabular}{|c|c|c|c|c|c|c|c|c|}
\hline \multirow[t]{2}{*}{ Variáveis } & \multicolumn{3}{|c|}{ Hospitalizações } & \multicolumn{3}{|c|}{ Prevalência de sibilância } & \multirow[t]{2}{*}{$\mathbf{R P}_{\text {robusto }}$} & \multirow[t]{2}{*}{ IC95\% } \\
\hline & $\mathrm{n}$ & $\%$ & IC95\% & $\mathrm{n}$ & $\%$ & IC95\% & & \\
\hline \multicolumn{9}{|l|}{ Nível 4} \\
\hline Padrão radiológico & & & & & & & $p=0,449$ & \\
\hline Infiltrado alveolar & 77 & 33,0 & $26,8-39,0$ & 42 & 54,5 & $43,3-65,8$ & 1,00 & \\
\hline $\begin{array}{l}\text { Infiltrado intersticial/ } \\
\text { hiperinsuflação } \\
\text { e/ou retificação arco }\end{array}$ & 21 & 9,0 & $5,3-12,7$ & 14 & 66,7 & $45,9-87,4$ & 1,10 & $0,86-1,40$ \\
\hline Eosinófilos (\%) & & & & & & & $p=0,784$ & \\
\hline$<4$ & 127 & 85,2 & $79,5-91,0$ & 77 & 60,6 & $52,0-69,2$ & 1,00 & \\
\hline$\geq 4$ & 22 & 14,8 & $9,0-20,5$ & 14 & 63,6 & $43,3-84,0$ & 1,05 & $0,74-1,48$ \\
\hline
\end{tabular}

IC95\%: intervalo de 95\% de confiança; RP: razão de prevalência.

Figura 1

Modelo final dos fatores associados à sibilância. Crianças Guarani no Sul e Sudeste do Brasil, 2007/2008.

\begin{tabular}{|l|l|}
\hline Nível 1 & $\begin{array}{l}\text { Região de residência } \\
\text { Sudeste: referência } \\
\text { Sul: } 1,19(0,96-1,48)\end{array}$ \\
\hline
\end{tabular}

\begin{tabular}{|c|c|c|}
\hline Nível 2 & $\begin{array}{l}\text { Faixa etária (meses) } \\
\text { 0-11: referência } \\
\text { 12-23: } 0,77(0,59-1,00) \\
\text { 24-35: } 0,63(0,40-0,99) \\
\text { 36-59: } 0,82(0,58-1,17)\end{array}$ & $\begin{array}{l}\text { Estação de internação } \\
\text { Verão: referência } \\
\text { Outono: } 1,58(1,05-2,40) \\
\text { Inverno: } 1,26(0,80-1,99) \\
\text { Primavera: } 1,52(0,99-2,35\end{array}$ \\
\hline
\end{tabular}

\begin{tabular}{|l|llll|}
\hline Nível 3 & Tosse & Febre & Taquipneia & Dispneia \\
Não: referência & Não: referência & Não: referência & Não: referência \\
Sim: $0,80(0,64-1,01)$ & Sim: $0,86(0,70-1,07)$ & Sim: $1,19(0,96-1,47)$ & Sim: $1,41(1,09-1,83)$ \\
& & & \\
& Tiragem & Estertor & Recusa alimentar & Batimento de asa do nariz \\
Não: referência & Não: referência & Não: referência & Não: referência \\
Sim: $1,42(1,16-1,73)$ & Sim: $1,43(1,09-1,87)$ & Sim: $0,54(0,23-1,28)$ & Sim: $0,44(0,15-1,31)$ \\
\hline
\end{tabular}

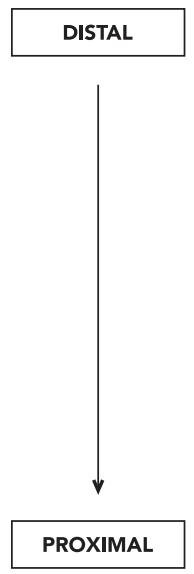

das crianças. A sibilância apresentou associação estatisticamente significativa com diagnóstico de IRA não pneumonia descrito no prontuário. Duas crianças hospitalizadas com sibilância evoluíram ao óbito (dados não tabulados).

\section{Discussão}

A elevada frequência de sibilância e de internações por essa causa é fenômeno mundial e constitui problema de saúde pública para todos os pa- íses, independente do grau de desenvolvimento 17,18,19. Nesse estudo, a sibilância esteve presente em mais da metade $(58,1 \%)$ das crianças Guarani hospitalizadas por IRAB. A prevalência de sibilância foi significativamente menor na faixa etária de 24 e 35 meses de idade e significativamente maior nas crianças hospitalizadas no outono e naquelas com registro em prontuário de dispneia, tiragem e estertor.

A prevalência de sibilância nas crianças Guarani hospitalizadas foi mais elevada no Sul $(65,3 \%)$ do que no Sudeste $(54,7 \%)$, ainda que 
Frequência de hospitalização e prevalência e razões de prevalência de sibilância segundo variáveis de diagnóstico, tratamento e desfecho.

\begin{tabular}{|c|c|c|c|c|c|c|c|c|}
\hline \multirow[t]{2}{*}{ Variáveis } & \multicolumn{3}{|c|}{ Hospitalizações } & \multicolumn{3}{|c|}{ Prevalência de sibilância } & \multirow{2}{*}{$\mathrm{RP}_{\text {robusto }}$} & \multirow[t]{2}{*}{ IC95\% } \\
\hline & $\mathbf{n}$ & $\%$ & IC95\% & $\mathbf{n}$ & $\%$ & IC95\% & & \\
\hline Hipótese diagnóstica hospitalar & & & & & & & $p=0,028$ & \\
\hline IRA pneumonia & 192 & 82,1 & $77,1-87,0$ & 106 & 55,2 & $48,1-62,3$ & 1,00 & \\
\hline IRA não pneumonia & 42 & 17,9 & $13,0-23,0$ & 30 & 71,4 & $57,6-85,2$ & 1,29 & $1,03-1,63$ \\
\hline Hipótese diagnóstica do revisor & & & & & & & $p=0,384$ & \\
\hline IRA pneumonia & 158 & 67,5 & $61,5-73,6$ & 95 & 60,1 & $52,4-67,8$ & 1,00 & \\
\hline IRA não pneumonia & 76 & 32,5 & $26,4-38,5$ & 41 & 53,9 & $42,6-65,2$ & 0,90 & $0,70-1,14$ \\
\hline Uso de antibiótico & & & & & & & $p=0,104$ & \\
\hline Não & 9 & 3,8 & $1,36-6,32$ & 7 & 77,8 & $50,4-105,1$ & 1,00 & \\
\hline Sim & 225 & 96,2 & $93,7-98,6$ & 129 & 57,3 & $50,8-63,8$ & 0,74 & $0,51-1,06$ \\
\hline Duração da internação (dias) & & & & & & & $p=0,616$ & \\
\hline $1-3$ & 33 & 14,1 & $9,61-18,6$ & 18 & 54,5 & $37,4-71,6$ & 1,00 & \\
\hline $4-7$ & 118 & 50,4 & $44,0-57,0$ & 67 & 56,8 & $47,8-65,8$ & 1,04 & $0,73-1,48$ \\
\hline $8-10$ & 37 & 15,8 & $11,1-20,5$ & 24 & 64,9 & $49,4-80,4$ & 1,19 & $0,80-1,76$ \\
\hline $11-15$ & 32 & 13,7 & $9,24-18,1$ & 19 & 59,4 & $42,2-76,5$ & 1,09 & $0,71-1,66$ \\
\hline $16-63$ & 14 & 6,0 & $2,92-9,04$ & 8 & 57,1 & $31,0-83,2$ & 1,05 & $0,60-1,82$ \\
\hline Reinternação da criança no mesmo episódio & & & & & & & $p=0,229$ & \\
\hline Não & 229 & 97,9 & $96,0-99,7$ & 135 & 59,0 & $52,5-65,4$ & 1,00 & \\
\hline $\operatorname{Sim}$ & 5 & 2,1 & $0,27-4,00$ & 1 & 20,0 & $15,3-55,3$ & 0,34 & $0,06-1,97$ \\
\hline Novos episódios de internação na mesma criança & & & & & & & $p=0,469$ & \\
\hline Não & 176 & 75,2 & $69,6-80,8$ & 100 & 56,8 & $49,4-74,6$ & 1,00 & \\
\hline Sim & 58 & 24,8 & $19,2-30,3$ & 36 & 62,1 & $49,5-74,6$ & 1,09 & $0,86-1,39$ \\
\hline
\end{tabular}

IC95\%: intervalo de 95\% de confiança; IRA: infecção respiratória aguda; RP: razão de prevalência.

essa diferença não tenha sido estatisticamente significativa. Esses resultados divergem dos reportados pela Pesquisa Nacional de Demografia e Saúde da Criança e da Mulher, na qual se verificou maior prevalência de sibilância referida na população infantil da Região Sudeste em relação ao Sul 19. Tendo em vista que o primeiro estudo se refere à prevalência de sibilância em crianças indígenas hospitalizadas e o segundo, à prevalência de sibilância referida em crianças da população geral, eventuais diferenças regionais podem decorrer de fatores ligados ao acesso a serviços de saúde, à qualidade da atenção e às exposições diferenciadas a risco ambientais, nutricionais, entre outros.

A idade mostrou-se inversamente relacionada à prevalência de sibilância nas crianças Guarani. A precocidade na apresentação de sibilância em crianças foi descrita por diversos autores em populações carentes atendidas em serviços públicos de saúde em cidades do Sul e Sudeste do Brasil 2,20,21. Em estudo em pacientes atendidos em unidades básicas de saúde em São Paulo, encontrou-se uma prevalência elevada de sibilância, com quase metade dos lactentes apresentando pelo menos um episódio de sibilância já no primeiro ano de vida 2 .

Observou-se que a prevalência de sibilância nos Guarani foi mais elevada nas internações ocorridas no outono, seguido da primavera. No Rio de Janeiro, estudos relataram maior incidência de doença causada por vírus sincicial respiratório (VSR) no período de outono e início do inverno, frequentemente associada à sibilância 22,23 . Na Região Sul, verifica-se padrão epidemiológico semelhante ao dos países de clima temperado, nos quais é maior a incidência de doença viral associada à sibilância no inverno e início da primavera 24 . As frequências elevadas de hospitalização Guarani com sibilância no outono e na primavera podem refletir diferenças nos padrões regionais de sazonalidade da doença.

Dispneia e tiragem estiveram significativamente associadas à sibilância nos Guarani. A dispneia associada à sibilância é considerada um dos critérios diagnósticos de bronquite viral 
aguda em lactentes e, levando-se em consideração outras doenças pertencentes à "síndrome sibilante", a asma é considerada o principal diagnóstico diferencial ${ }^{3}$. Por outro lado, a pneumonia bacteriana apresenta manifestações clínicas semelhantes à bronquite viral aguda, asma e outras afecções respiratórias, podendo também apresentar dispneia e tiragem, mas sem sibilância. Os sinais clínicos preconizados para a classificação da gravidade de pneumonia em crianças $<5$ anos incluem a presença de tiragem, na qual a subcostal é a que está mais associada à gravidade da pneumonia, embora outros tipos de retração possam estar presentes 25 .

A presença de estertor subcrepitante associado à sibilância e/ou roncos na ausculta pulmonar em crianças com suspeita de doença respiratória aguda é sugestiva de bronquite viral aguda ${ }^{3}$. Nos prontuários hospitalares das crianças Guarani, o registro de estertor nem sempre estava acompanhado de especificação de suas características, permitindo supor que, em parte, ele pudesse se caracterizar como subcrepitante. Apesar disso, $55,2 \%$ das crianças com hipótese de pneumonia registrada em prontuário apresentaram sibilância, bem como a prevalência de sibilância foi de $54,5 \%$ nas crianças com registro de infiltrado alveolar na radiografia de tórax. Esses achados sugerem a concomitância da circulação de agentes virais e bacterianos na população de estudo 11,25,26,27,28, ou ainda, a possível ocorrência de pneumonia bacteriana em crianças portadoras de asma 5 .

Em decorrência do uso de dados secundários e a consequente indisponibilidade de informação sobre história familiar de atopia, não é possível afirmar o fenótipo de sibilância nas crianças Guarani. No entanto, a alta frequência de sibilância em $<3$ anos e sua elevada prevalência em crianças < 12 meses sugerem um fenótipo de sibilância persistente de início precoce, cuja recorrência é deflagrada principalmente por infecções respiratórias agudas virais 5 . A presença de sibilância frequente durante os três primeiros anos de vida associada a um fator de risco maior (história parental de asma ou pessoal de eczema) ou dois de três fatores de risco menores (eosinofilia $\geq 4 \%$, sibilância na ausência de infecções de vias aéreas superiores e rinite alérgica) indica risco elevado do lactente ser asmático 29 . A eosinofilia ( $\geq 4 \%$ ) não se mostrou significativamente associada à sibilância nas crianças Guarani, o que não exclui a possibilidade de inflamação eosinofílica em tecido pulmonar 30 , mas reforça a hipótese de determinação ambiental.

Verificou-se que 57,3\% das crianças Guarani que receberam antibiótico durante a hospitalização possuíam registro de sibilância no prontuá- rio. Estudo realizado com crianças hospitalizadas com IRAB no Rio de Janeiro mostrou que o percentual de uso de antibióticos iniciados no setor de emergência entre pacientes VSR positivos foi alto e maior do que o encontrado nos pacientes VSR negativos, o que pode ser atribuído a pouca especificidade dos dados clínicos-radiológicos para definição da etiologia das IRAB nessa faixa etária 28,31 . Além disso, existe a possibilidade dos pacientes sibilantes terem complicado com pneumonia bacteriana 25,28.

A permanência hospitalar foi de até dez dias em $80,3 \%$ das hospitalizações e a prevalência de sibilância foi maior no período de oito a dez dias (64,9\%). Na Europa, lactentes admitidos no hospital por bronquite viral aguda apresentaram média de permanência de oito a nove dias 32 . Contudo, em nosso meio, as condições socioeconômicas desfavoráveis muitas vezes determinam internações prolongadas, não necessariamente relacionadas à gravidade do caso 31 .

O desfecho da hospitalização foi favorável na maioria dos casos. Foram registrados dois óbitos, apenas em crianças com sibilância e, um paciente reinternou no mesmo episódio com sibilância. Nos pacientes que apresentaram novos episódios de internação, a prevalência de sibilância foi $62,1 \%$, enquanto nos que não reinternaram por novos episódios, essa prevalência foi de $56,8 \%$, sem diferença estatisticamente significativa. Estudos sugerem que a sibilância recorrente tem maior prevalência e gravidade em países em desenvolvimento do que em países desenvolvidos 33,34 , atribuindo tal fato a fatores ambientais e às más condições socioeconômicas das populações dessas localidades 2 .

Algumas limitações devem ser consideradas na interpretação dos resultados desse estudo. Por circunstâncias relacionadas ao desenho epidemiológico de estudo caso-controle, que gerou os dados desse trabalho, a prevalência de sibilância foi estudada somente na população de crianças hospitalizadas por IRAB, que possivelmente apresenta características diferenciadas em relação ao conjunto das crianças indígenas menores de cinco anos residentes nas aldeias. Essa opção metodológica poderia resultar em superestimação das prevalências de sibilância. Por outro lado, o reconhecimento dos fatores associados à sibilância no grupo hospitalizado contribuiu para a identificação de padrões sazonais de adoecimento, grupos etários de maior risco e condições clínicas indicativas de gravidade.

Outro aspecto relevante se refere à fonte secundária dos dados para o estudo - os prontuários hospitalares. Foram revisados prontuários em 49 hospitais em cinco estados das regiões Sul e Sudeste, com níveis diversos de organização e 
complexidade da atenção. Desta forma, verificou-se expressiva variação na qualidade dos registros hospitalares e na solicitação de exames complementares.

Considerou-se mais provável que a ausência de registro de um sinal ou sintoma no prontuário decorresse da ausência do mesmo na criança por ocasião da avaliação hospitalar do que da omissão do seu registro pelo profissional assistente. Assim, assumiu-se a ausência do registro em prontuário como ausência do sinal ou sintoma na criança. Essa decisão poderia desconsiderar a presença de sinais e sintomas quando ocorresse falha de registro no prontuário, o que seria mais provável nos casos menos graves e nos hospitais de menor porte e estrutura. Tal fato poderia resultar em viés nas análises sobre associação com sibilância no grupo. Contudo, os sinais clínicos mais comuns na IRAB apresentaram elevada frequência de positividade, enfraquecendo a hipótese de viés de informação. Soma-se a isso, a coerência dos resultados desse estudo com os descritos na literatura para crianças não indígenas em condições desfavoráveis de vida e saúde.

Com relação aos exames complementares, a radiografia de tórax e o hemograma completo são sempre indicados como auxílio diagnóstico nos casos de pneumonia que necessitam internação 25,28 . Verificou-se que $63,7 \%$ e $43,2 \%$ das crianças possuíam registro de resultados de hemograma e radiografia de tórax, respectivamente. O poder do estudo e as perdas de dados relativos aos exames complementares podem ter resultado na ausência de associação dos padrões radiológicos e de eosinofilia com sibilância.

A informação hospitalar tem como parte essencial o prontuário médico, que consiste em importante ferramenta de registro do processo e resultado dos cuidados prestados, além de ser considerado um instrumento de educação e pesquisa. Portanto, a precariedade das informações relativas ao paciente reflete certo grau de dificuldade na prestação do cuidado e impossibilita análises mais acuradas de aspectos fundamentais da investigação sobre determinantes da saúde $35,36,37$.

\section{Considerações finais}

A elevada prevalência de sibilância entre crianças indígenas Guarani hospitalizadas por IRAB, particularmente em menores de um ano, revela uma elevada carga de morbidade relacionada à sibilância nessa população. O padrão verificado sugere o fenótipo de sibilância persistente de início precoce nas crianças Guarani, o que indica a necessidade de investimentos na prevenção e controle das infecções respiratórias agudas virais e na redução da exposição continuada a agentes inalantes do ambiente. A ausência de exames complementares e uso maciço de antibióticos, possivelmente também em infecções virais, apontam para a necessidade de implementar e monitorar ações que visem à melhoria no diagnóstico e manejo desses agravos nessa população, tanto em ambiente comunitário quanto em ambiente hospitalar, tal como recomendado pela Organização Mundial da Saúde (OMS) em protocolos de diagnóstico e manejo das IRAB nos hospitais de pequeno porte ${ }^{38}$.

Indica-se a necessidade de melhorias nos registros hospitalares como meio de permitir o aprimoramento da qualidade da assistência ao paciente e a continuidade do cuidado pela equipe multiprofissional, bem como possibilitar monitoramento epidemiológico das hospitalizações e suas causas. Considera-se útil e oportuna a realização de mais estudos que contribuam para o conhecimento da epidemiologia das IRA que cursam com sibilância em crianças indígenas Guarani, a fim de subsidiar programas de tratamento, prevenção e promoção da saúde infantil. 


\section{Resumen}

Se realizó un estudio de la prevalencia de sibilancia en niños indígenas guaranies hospitalizados por infección respiratoria aguda en el sur y sureste de Brasil, recogidos por el sistema de vigilancia desplegado en poblados indígenas entre mayo de 2007 y junio de 2008. Los datos fueron obtenidos mediante la revisión de los registros hospitalarios. Se estimaran las razones de prevalencia de sibilancia bruta y ajustada, de acuerdo con las categorías de las variables explicativas, utilizando la regresión de Poisson con varianza robusta. La prevalencia de sibilancia fue de un 58,1\%. Se mantuvo significativamente asociada con la sibilancia en el modelo final: rango de edad, asociado inversamente (0-11 meses: referencia.; 24-35 meses: 0,63, IC95\%: 0,40-0,99); hospitalización en otoño (verano: referencia; otoño: 1,58 , IC95\%: 1,05-2,40); disnea (1,41, IC95\%: 1,09-1,83); tiraje (1,42, IC95\%: 1,16-1,73); estertores (1,43, IC95\%: 1,091,87). Los resultados revelan una alta carga de morbilidad relacionada la sibilancia y sugieren el fenotipo de sibilancia persistente de inicio precoz, relacionada con la recurrencia de infección respiratoria viral.

Ruidos Respiratorios; Neumonía; Indios

Sudamericanos; Niño; Prevalencia

\section{Referências}

1. Martinez FD, Wright AL, Taussig LM, Holberg CJ, Halonen M, Morgan WJ. Asthma and wheezing in the first six years of life. The Group Health Medical Associates. N Engl J Med 1995; 332:133-8.

2. Bianca ACCD, Wandalsen GF, Mallol J, Solé D. Prevalência e gravidade da sibilância no primeiro ano de vida. J Bras Pneumol 2010; 36:402-9.

3. Sant'Anna CC, D'Elia C. Bronquiolite. In: Benguigui Y, Antuñano FJL, Schumunis G, Yunes J, organizadores. Infecções respiratórias em crianças. Brasília: Organização Pan-Americana da Saúde/ Organização Mundial da Saúde; 1998. p. 263-81. (Série HCT/AIEPI-1).

4. Bianca ACCD, Wandalsen GF, Solé D. Lactente sibilante: prevalência e fatores de risco. Rev Bras Alergia Imunopatol 2010; 33:43-50.

5. Global Initiative for Asthma. Global strategy for asthma management and prevention 2012 (update). Bethesda: Global Initiative for Asthma; 2013.

\section{Colaboradores}

P. G. Souza, A. M. Cardoso e C. C. Sant'Anna participaram da concepção do projeto, análise e interpretação dos resultados, redação do artigo e aprovação final da versão publicada.

\section{Agradecimentos}

Os autores agradecem às equipes técnicas das Assessorias de Saúde Indígena da FUNASA responsáveis pela saúde indígena Guarani no Sul e Sudeste do Brasil na ocasião de coleta dos dados; às Equipes Multidisciplinares de Saúde Indígena, particularmente aos enfermeiros e equipe de enfermagem e, acima de tudo, aos Guarani, que apoiaram e acreditaram no trabalho como uma contribuição potencial para a saúde de seus filhos. O estudo foi financiado pela FAPERJ (E26/102.818/2008) e CNPq (processos no 402522/2008-7 e no $40.9677 / 2006-0$ ).
6. Montenegro RA, Stephens C. Indigenous health in Latin America and the Caribbean. Lancet 2006; 367:1859-69.

7. Cardoso AM, Coimbra Jr. CEA, Guimarães FT. Morbidade hospitalar indígena Guarani no Sul e Sudeste do Brasil. Rev Bras Epidemiol 2010; 13:21-34.

8. Cardoso AM, Coimbra Jr. CEA, Barreto CTG, Werneck GL, Santos RV. Mortality among Guarani Indians in Southeastern and Southern Brazil. Cad Saúde Pública 2011; 27 Suppl 2:S222-36.

9. Lunardi R, Santos RV, Coimbra Jr. CEA. Morbidade hospitalar de indígenas Xavante, Mato Grosso, Brasil (2000-2002). Rev Bras Epidemiol 2007; 10:441-52.

10. Orellana JDY, Basta PC, Santos RV, Coimbra Jr. CEA. Morbidade hospitalar em crianças indígenas Suruí menores de dez anos, Rondônia, Brasil: 2000 a 2004. Rev Bras Saúde Matern Infant 2007; 7:281-7. 
11. Portela MFP, Sant'Anna CC, Campos Júnior D. Pneumonia em crianças e adolescentes indígenas. Pulmão RJ 2005; 14:283-8.

12. Gracey M, King M. Indigenous health part 1: determinants and disease patterns. Lancet 2009; 374:65-75.

13. King M, Smith A, Gracey M. Indigenous health part 2: the underlying causes of the health gap. Lancet 2009; 374:76-85.

14. Cardoso AM, Coimbra Jr. CEA, Werneck GL. Risk factors for hospital admission due to acute lower respiratory tract infection in Guarani indigenous children in Southern Brazil: a population-based case-control study. Trop Med Int Health 2013; 18:596-607.

15. Cardoso AM. Doença respiratória aguda em indígenas Guarani no Sul e Sudeste do Brasil [Tese de Doutorado]. Rio de Janeiro: Escola Nacional de Saúde Pública Sergio Arouca, Fundação Oswaldo Cruz; 2010.

16. Barros AJ, Hirakata VN. Alternatives for logistic regression in cross-sectional studies: an empirical comparison of models that directly estimate the prevalence ratio. BMC Med Res Methodol 2003; 3:21.

17. Beasley R, Ellwood P, Asher I. International patterns of the prevalence of pediatric asthma the ISAAC program. Pediatr Clin North Am 2003; 50:539-53.

18. World Health Organization. Asthma. http://www. who.int/respiratory/asthma/en/index.html (acessado em 15/Jun/2013).

19. Centro Brasileiro de Análise e Planejamento, Ministério da Saúde. Pesquisa Nacional de Demografia e Saúde da Criança e da Mulher - PNDS 2006: dimensões do processo reprodutivo e da saúde da criança. Brasília: Ministério da Saúde; 2009. (Série G. Estatística e Informação em Saúde).

20. Lima JAB, Fisher GB, Sarria EE, Mattiello R, Solé D. Prevalência e fatores de risco para sibilância no primeiro ano de vida. J Bras Pneumol 2010;36: 525-31.

21. Chong Neto HJ, Rosário NA, Grasselli EA, Silva FC, Bojarski LFM, Rosário CS, et al. Sibilância recorrente em lactentes: mudanças epidemiológicas. J Pediatr (Rio J.) 2011; 87:547-50.

22. Nascimento JP, Siqueira MM, Sutmoller F, Krawczuk MM, Farias V, Ferreira V, et al. Longitudinal study of acute respiratory diseases in Rio de Janeiro: occurrence of respiratory viruses during four consecutive years. Rev Inst Med Trop São Paulo 1991; 33:287-96.

23. Sutmoller F, Ferro ZP, Asensi MD, Ferreira V, Mazzei IS, Cunha BL. Etiology of acute respiratory tract infections among children in a combined community and hospital study in Rio de Janeiro. Clin Infect Dis 1995; 20:854-60.

24. Straliotto SM. Prevalência de vírus respiratórios em pacientes pediátricos em Porto Alegre [Dissertação de Mestrado]. Porto Alegre: Faculdade de Ciências Médicas de Porto Alegre; 1995.
25. Sociedade Brasileira de Pneumologia e Tisiologia. Diretrizes brasileiras em pneumonia adquirida na comunidade em pediatria - 2007. J Bras Pneumol 2007; 33 Suppl 1:S31-50.

26. Pereira JC, Escuder MM. Susceptibility of asthmatic children to respiratory infection. Rev Saúde Pública 1997; 31:441-7.

27. Hall CB, Hall WJ, Speers DM. Clinical and physiological manifestations of bronchiolitis and pneumonia. Am J Dis Child 1979; 133:798-802.

28. Izadnegahdar R, Cohen AL, Klugman KP, Qazi AS. Childhood pneumonia in developing countries. Lancet Respir Med 2013; 1:574-84.

29. Castro-Rodriguez JA, Holberg CJ, Wright AL, Martinez FD. A clinical index to define risk of asthma in young children with recurrent wheezing. Am J Respir Crit Care Med 2000; 162:1403-6.

30. Medeiros D, Silva AR, Rizzo JA, Motta ME, Oliveira FHB, Sarinho ESC. Nível sérico de IgE total em alergia respiratória: estudo em pacientes com alto risco de infecção por helmintos. J Pediatr (Rio J.) 2006; 82:255-9.

31. D’Elia C, Siqueira MM, Portes SA, Santa'Anna CC. Infecções do trato respiratório inferior pelo vírus sincicial respiratório em crianças hospitalizadas menores de um ano de idade. Rev Soc Bras Med Trop 2005; 38:7-10.

32. Behrendt CE, Decker MD, Burch DJ, Watson PH. International variation in the management of infants hospitalized with respiratory syncytial virus. International RSV Study Group. Eur J Pediatr 1998; 157:215-20.

33. Mallol J, Andrade R, Auger F, Rodríguez J, Alvarado $R$, Figueroa L. Wheezing during the first year of life in infants from low-income population: a descriptive study. Allergol Immunopathol (Madr) 2005; 33:257-63.

34. Schwartz J, Gold D, Dockery DW, Weiss ST, Speizer FE. Predictors of asthma and persistent wheeze in a national sample of children in the United States. Association with social class, perinatal events, and race. Am Rev Respir Dis 1990; 142:555-62.

35. Escosteguy CC, Portela MC, Medronho RA, Vasconcellos MTL. O Sistema de Informações Hospitalares e a assistência ao infarto agudo do miocárdio. Rev Saúde Pública 2002; 36:491-9.

36. Mesquita AMO, Deslandes SF. A construção dos prontuários como expressão da prática dos profissionais de saúde. Saúde Soc 2010; 19:664-73.

37. Pavão ALB, Andrade D, Mendes W, Martins M, Travassos C. Estudo de incidência de eventos adversos hospitalares, Rio de Janeiro, Brasil: avaliação da qualidade do prontuário do paciente. Rev Bras Epidemiol 2011; 14:651-61.

38. Ministério da Saúde. Manual de normas para controle e assistência das infecções respiratórias agudas. 3ạ Ed. Brasília: Ministério da Saúde; 1993.

Recebido em 27/Ago/2013

Versão final reapresentada em 27/Dez/2013

Aprovado em 06/Jan/2014 
Evolution of the quality of mortality data in males. Brazil, 1980-2010. 